\title{
Beyond Detection: Novel Applications for PET Imaging to Guide Cancer Therapy
}

A termine the behavior of cancer. In describing the mechanistic aspects of cancer growth, Hanahan et al. have detailed these functional changes - such as altered metabolism, proliferation, invasiveness, and metastatic potentialthat produce a chaotic but complex situation (1). Altered perfusion in a solid tumor, as a result of lagging blood supply disproportionate to cellular proliferation and energy metabolism, results in hypoxia, the presence of which is not related to tumor size, stage, or histologic type. Hypoxia itself

\section{See page 973}

can induce additional changes in the biology of cancer cells that will result in aggressive phenotype and increased metastatic potential due to several hypoxiarelated genes that are mediated primarily via the transcription factor hypoxiainducible factor (HIFl $\alpha)$ (2). There is induction of vascular endothelial growth factor, glucose transporter, hexokinase, and selection of p53 mutant cells, all of which result in downstream changes.

Even though the negative association of tumor hypoxia and clinical prognosis has been known for a long time, the lack of sensitive noninvasive methods has slowed progress in the routine clinical application of hypoxia

Received Mar. 14, 2007; revision accepted Mar. 20, 2007.

For correspondence or reprints contact: Joseph Rajendran, MD, Division of Nuclear Medicine, Department of Radiology, Box 356113, University of Washington, Seattle, WA 98195.

E-mail: rajan@u.washington.edu

COPYRIGHT (c) 2007 by the Society of Nuclear Medicine, Inc.

DOI: 10.2967/jnumed.107.039768 evaluation to cancer care. Simple clinical predictors, such as tumor size and hemoglobin concentration, have been tried but are of limited accuracy in predicting tumor hypoxia (3). Early attempts to overcome hypoxia-mediated treatment resistance used more radiation, hyperbaric oxygen therapy (along with radiotherapy), or low oxygen enhancement ratio, but with limited success $(4,5)$. Hypoxia-selective radiosensitizers, such as misonidazole, were tried in clinical trials but were limited by toxicity and minimal success.

The unique microenvironment associated with hypoxic tumors makes tumor hypoxia an attractive target for hypoxia-activated prodrugs (6). Although focal hypoxia can be targeted with additional radiation using intensitymodulated radiotherapy (IMRT) to selectively boost the overall dose (7), more diffuse hypoxia will benefit from systemic hypoxic cell toxins or sensitizers that specifically accumulate in hypoxic tissue. Newer hypoxia-activated prodrugs with less toxicity and more effectiveness have been synthesized and are undergoing clinical trials (8). The most widely tested hypoxic-cell cytotoxin is tirapazamine (TPZ). In addition to direct cytotoxic effects on hypoxic cells, TPZ exhibits synergistic toxicity with radiation and chemotherapy $(9,10)$. Earlier as well as more recent results suggest overwhelmingly that TPZ is more effective in the presence of tumor hypoxia $(11,12)$.

The availability of novel hypoxiaspecific prodrugs makes identification of hypoxia in tumors more important. Oxygen electrode measurements, which provided the basis for much of our early understanding of tumor hypoxia, are limited by practical challenges, such as invasiveness and tumor accessibility. There is spatial as well as temporal heterogeneity, both in the distribution of hypoxia and the hypoxia response, which require a noninvasive in vivo assay that can be performed in a repeated fashion. Molecular imaging has made rapid strides that go beyond the clinical applications of FDG PET, to probe multiple aspects of tumor biology (13-15). The real power of molecular imaging goes beyond diagnosis by identifying different biologic processes in a tumor using tracers that characterize both genotypic and phenotypic signatures. $(16,17)$. PET with several hypoxia-specific tracers has the ability to quantify hypoxia and provides a basis for rational patient selection and for guiding treatment.

After extensive validation, fluoromisonidazole (FMISO) PET remains as one of the most common PET hypoxia tracers. Rajendran et al. have shown the utility of pretherapy ${ }^{18} \mathrm{~F}$ FMISO PET in predicting survival in a cohort of 73 patients with head and neck cancer (18). This tracer has several desirable characteristics for an ideal clinical hypoxia assay: simple, noninvasive, rapid and easy to use with consistency between laboratories, and the ability to quantify with available PET scanner technology.

In an important study demonstrating the use of PET to direct hypoxiaspecific treatment, Rischin et al. showed that uptake of ${ }^{18} \mathrm{~F}$-FMISO by PET predicted the greater effectiveness of TPZ (11). In this study, only patients with ${ }^{18} \mathrm{~F}$-FMISO uptake benefited from the addition of TPZ to radiotherapy for head and neck cancer. Even though TPZ has minimal side effects (by no means negligible), some of the known side effects, such as muscle cramps, can be troublesome so as to warrant discontinuation of the drug. Therefore, it is important to identify patients with 
significant hypoxia in tumors that can benefit from TPZ or similar drugs rather than using such drugs on all patients regardless of the presence of hypoxia.

In this issue of The Journal of $\mathrm{Nu}$ clear Medicine, Beck et al. present a study on the role of another PET hypoxia agent in predicting response to TPZ in an animal model (19). The authors used ${ }^{18} \mathrm{~F}$-fluoroazamycin arabinoside ( $\left.{ }^{18} \mathrm{~F}-\mathrm{FAZA}\right)$, a PET hypoxia tracer with properties similar to those of ${ }^{18} \mathrm{~F}$-FMISO. In the Beck study, ${ }^{18} \mathrm{~F}$ FAZA uptake by PET predicted rapid tumor growth as well as a benefit from adding TPZ to radiotherapy in the animal model. Like the Rischin article on humans $(11,12)$, Beck et al. have demonstrated, using an animal model and a different hypoxia radiopharmaceutical, that PET can be used to select hypoxia-specific treatment.

The utility of hypoxia imaging in directing cancer therapy could go beyond TPZ, as several novel and promising drugs are now available and are being evaluated. One such example is efaproxiral, an allosteric modifier of hemoglobin, which has the potential to increase tissue oxygenation of hypoxic tumor cells by reducing the oxygen-binding capacity of hemoglobin. It has shown promise in early clinical trials (20). To be effective, successful cancer therapy should target many of the complex and interrelated pathways involved in the growth of cancer rather than targeting only one aspect of the microenvironment such as hypoxia. The real power of a noninvasive molecular imaging method, such as hypoxia imaging in clinical decision-making processes, will be seen when multiple probes are used to characterize the various biologic processes of the tumor in a given patient. Information from molecular imaging will provide the oncologist with the ability to tailor treatment for the individual patient that is based on molecular pathways of the tumor and its microenvironment, such as hypoxia. Future clinical trials, with appropriate study design and regulatory guidance, will need to examine prospectively the use of imaging to help select cancer treatment. This is an important paradigm shift for PET, moving beyond detection in the direction of treatment selection.

\section{Joseph G. Rajendran David A. Mankoff University of Washington Seattle, Washington}

\section{REFERENCES}

1. Hanahan D, Weinberg RA. The hallmarks of cancer. Cell. 2000;100:57-70.

2. Semenza GL. Targeting HIF-1 for cancer therapy. Nat Rev Cancer. 2003;3:721-732.

3. Rajendran JG, Ng P, Peterson LM, et al. F-18 FMISO PET tumor hypoxia imaging: investigating the tumor volume-hypoxia connection [abstract]. J Nucl Med. 2003;44(suppl):376P.

4. Fowler JF. Eighth Annual Juan del Regato lecture. Chemical modifiers of radiosensitivity: theory and reality-a review. Int J Radiat Oncol Biol Phys. 1985;11:665-674.

5. Overgaard J, Horsman MR. Modification of hypoxia-induced radioresistance in tumors by the use of oxygen and sensitizers. Semin Radiat Oncol. 1996;6:10-21.

6. Brown JM. The hypoxic cell: a target for selective cancer therapy-Eighteenth Bruce F. Cain Memorial Award lecture. Cancer Res. 1999;59:58635870.

7. Rajendran JG, Hendrickson KR, Spence AM, Muzi M, Krohn KA, Mankoff DA. Hypoxia imagingdirected radiation treatment planning. Eur J Nucl Med Mol Imaging. 2006;33(suppl 13):44-53.

8. Denny WA. Prospects for hypoxia-activated anticancer drugs. Curr Med Chem Anticancer Agents. 2004;4:395-399.
9. von Pawel J, von Roemeling R, Gatzemeier U, et al. Tirapazamine plus cisplatin versus cisplatin in advanced non-small-cell lung cancer: a report of the international CATAPULT I study groupCisplatin and Tirapazamine in Subjects with Advanced Previously Untreated Non-Small-Cell Lung Tumors. J Clin Oncol. 2000;18:1351-1359.

10. Brown JM. Exploiting the hypoxic cancer cell: mechanisms and therapeutic strategies. Mol Med Today. 2000;6:157-162.

11. Rischin D, Hicks RJ, Fisher R, et al. Prognostic significance of $\left[{ }^{18} \mathrm{~F}\right]$-misonidazole positron emission tomography-detected tumor hypoxia in patients with advanced head and neck cancer randomly assigned to chemoradiation with or without tirapazamine: a substudy of Trans-Tasman Radiation Oncology Group Study 98.02. J Clin Oncol. 2006;24:2098-2104.

12. Rischin D, Peters L, Hicks R, et al. Phase I trial of concurrent tirapazamine, cisplatin, and radiotherapy in patients with advanced head and neck cancer. J Clin Oncol. 2001;19:535-542.

13. Rajendran JG, Wilson DC, Conrad EU, et al. $\left[{ }^{18} \mathrm{~F}\right]$ FMISO and $\left[{ }^{18} \mathrm{~F}\right]$ FDG PET imaging in soft tissue sarcomas: correlation of hypoxia, metabolism and VEGF expression. Eur J Nucl Med Mol Imaging. 2003;30:695-704.

14. Mankoff DA, Shields AF, Krohn KA. PET imaging of cellular proliferation. Radiol Clin North Am. 2005;43:153-167.

15. Linden HM, Stekhova SA, Link JM, et al. Quantitative fluoroestradiol positron emission tomography imaging predicts response to endocrine treatment in breast cancer. J Clin Oncol. 2006;24: 2793-2799.

16. Chapman JD, Bradley JD, Eary JF, et al. Molecular (functional) imaging for radiotherapy applications: an RTOG symposium. Int J Radiat Oncol Biol Phys. 2003;55:294-301.

17. Rajendran JG, Mankoff DA, O'Sullivan F, et al. Hypoxia and glucose metabolism in malignant tumors: evaluation by $\left[{ }^{18} \mathrm{~F}\right]$ fluoromisonidazole and $\left[{ }^{18} \mathrm{~F}\right]$ fluorodeoxyglucose positron emission tomography imaging. Clin Cancer Res. 2004;10:22452252.

18. Rajendran JG, Schwartz DL, O'Sullivan J, et al. Tumor hypoxia imaging with [F-18] fluoromisonidazole positron emission tomography in head and neck cancer. Clin Cancer Res. 2006;12:54355441.

19. Beck R, Röper B, Carlsen JM, et al. Pretreatment ${ }^{18}$ F-FAZA PET predicts success of hypoxia-directed radiochemotherapy using tirapazamine. $\mathrm{J} \mathrm{Nucl} \mathrm{Med}$. 2007;48:973-980.

20. Suh JH, Stea B, Nabid A, et al. Phase III study of efaproxiral as an adjunct to whole-brain radiation therapy for brain metastases. J Clin Oncol. 2006;24: 106-114. 